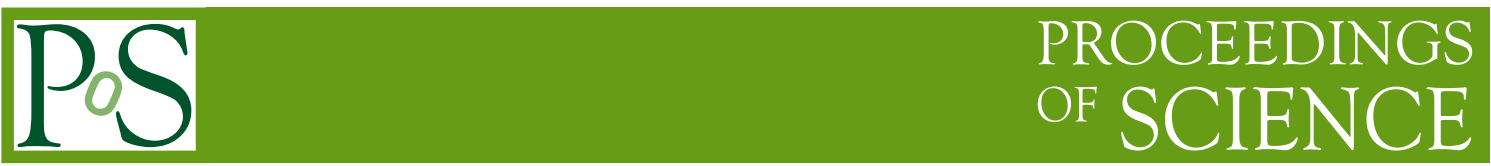

\title{
Neutrino CP Violation with the ESS $v$ SB project
}

\author{
Marcos Dracos ${ }^{* \dagger}$ \\ IPHC, Université de Strasbourg, CNRS/IN2P3, F-67037 Strasbourg, France \\ E-mail: marcos.dracosein2p3.fr
}

\begin{abstract}
After measuring in 2012 a relatively large value of the neutrino mixing angle $\theta_{13}$, the door is now open to observe for the first time a possible $\mathrm{CP}$ violation in the leptonic sector. The measured value of $\theta_{13}$ also privileges the 2 nd oscillation maximum for the discovery of $\mathrm{CP}$ violation instead of the usually used 1st oscillation maximum. The sensitivity at this 2 nd oscillation maximum is about three times higher than for the 1st oscillation maximum inducing a lower influence of systematic errors. Going to the 2nd oscillation maximum necessitates a very intense neutrino beam with the appropriate energy. The world's most intense pulsed spallation neutron source, the European Spallation Source, will have a proton linac with $5 \mathrm{MW}$ power and $2 \mathrm{GeV}$ energy. This linac, under construction, also has the potential to become the proton driver of the world's most intense neutrino beam with very high potential to discover a neutrino $\mathrm{CP}$ violation. The physics performance of that neutrino Super Beam in conjunction with a megaton underground Water Cherenkov neutrino detector installed at a distance of about $500 \mathrm{~km}$ from ESS has been evaluated. The choice of such detector will extent the physics program to proton-decay, atmospheric neutrinos and astrophysics searches. In addition to neutrinos, this facility will also produce at the same time a copious number of muons which could be used by other challenging projects. The ESS neutron facility will be fully ready by 2023 at which moment the upgrades for the neutrino facility could start. This project is now supported by the COST Action CA15139 "Combining forces for a novel European facility for neutrino-antineutrino symmetry-violation discovery" (EuroNuNet).

Keywords: ESSnuSB, neutrino, CP violation, neutrino oscillations, ESS, COST, EuroNuNet.
\end{abstract}

EPS-HEP 2017, European Physical Society conference on High Energy Physics

5-12 July 2017

Venice, Italy

\footnotetext{
* Speaker.

${ }^{\dagger}$ On behalf of the ESS $v$ SB project.
} 


\section{Introduction}

One important question remaining unanswered in cosmology is why matter has dominated antimatter. Sources of CP violation, producing an asymmetry between matter and antimatter, have been found in the hadronic sector, but by far not enough to explain the antimatter disappearance. After the measurement of the last neutrino oscillation angle $\theta_{13}$, found to be relatively large, all conditions are now met to observe a $\mathrm{CP}$ violation in the leptonic sector by studying the oscillation $v_{\mu} \rightarrow v_{e}$. If the $\delta_{C P}$ parameter is found far from $0^{\circ}$ and $180^{\circ}$, the asymmetry between neutrinos and antineutrinos could, under some assumptions, explain the matter-antimatter asymmetry in the Univers [1].

For the observation of CP Violation in the neutrino sector new next generation accelerator neutrino projects are in preparation or proposed. These projects rely on very intense neutrino beams using MW class proton drivers. The physics performance of these projects strongly depends on the systematic uncertainties which could be achieved by the moment of the operation of the relating experiments. One of these projects, the only one expected to be based in Europe, ESSvSB [2], proposes to use the very powerful proton linac, under construction, of the European Spallation Source (ESS) [3] to produce a high intensity muon neutrino beam, which could be sent towards a megaton Water Cherenkov detector located at a distance of about $500 \mathrm{~km}$ away. This distance corresponds to the position of the second oscillation maximum. Placing the far detector at the second oscillation maximum procures enhanced sensitivity to observe a possible CP Violation with less sensitivity to systematic errors [4], compared to the first oscillation maximum.

\section{The ESS $v$ SB Neutrino Facility}

The ESS neutron facility is under construction in Lund, Sweden, since 2014. The whole installation will be fully operational by 2023 . This facility will use a very powerful proton linac (Fig. 1) with an average beam power of $5 \mathrm{MW}$ and a duty cycle of only $4 \%$ (pulse duration of $2.86 \mathrm{~ms}$ ). By doubling the pulse frequency from $14 \mathrm{~Hz}$ to $28 \mathrm{~Hz}$, this facility could be used at the same time to produce neutrons and neutrinos by alternating the pulses between the two facilities. The number of protons on target per year would be for each facility of the order of $2.7 \times 10^{23}$. The proton kinetic energy is $2.0 \mathrm{GeV}$ and it could be go up to $3.6 \mathrm{GeV}$ in future upgrades using empty space left in purpose in the linac tunnel.

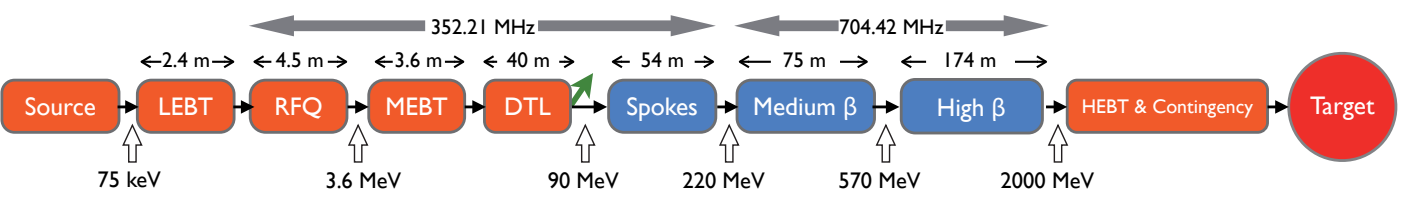

Figure 1: Schematic view of the ESS proton linac.

In order to shorten the proton pulses to few $\mu \mathrm{s}$, needed for technical reasons by the neutrino facility, $\mathrm{H}^{-}$have to be injected and accelerated in the linac. The $\mathrm{H}^{-}$have to be injected in an accumulation ring of a circumference of about $400 \mathrm{~m}$ with an electron stripping at the entrance of the ring. The proton beam extracted from the accumulator can be sent to a target followed by a 
hadronic collector to produce muon neutrinos from pion decays. A single classical magnetic horn can be used for the hadron focusing. The necessary magnetic field inside the horn, is produced by a very high pulsed current $(\sim 350 \mathrm{kA})$ using a specific pulse generator. The horn and the integrated target can be placed in a well shielded target station placed just after the accumulation ring. Preliminary studies showed that a short decay tunnel of $\sim 25 \mathrm{~m}$ length could be long enough to leave the pions decay to muons and short enough to avoid too many muon decays which would produce electron neutrinos and thus pollute the primary muon neutrino beam. At the end of the decay tunnel a beam dump has to be installed to stop all remaining particles. Fig. 2 presents a schematic view of the ESS neutron and neutrino facility.

The high power proton beam shock on the target, which could produce violent vibrations and power dissipation problems, could be mitigated be using four identical target/horn systems which could be pulsed alternatively. A beam switchyard can be used at the exit of the accumulator to distribute the beam to the four systems. This system has already been proposed and studied by the FP7 Design Study EUROv [5, 6].

In order to monitor the still unoscillated neutrino beam and to measure the relevant neutrino cross-sections, a near detector can be installed at about $500 \mathrm{~m}$ from the neutrino target. Studies are under way to find the best near detector configuration to do both tasks, able to detect muon neutrinos on one side and detect electron neutrinos with a very good muon neutrino rejection on the other side.

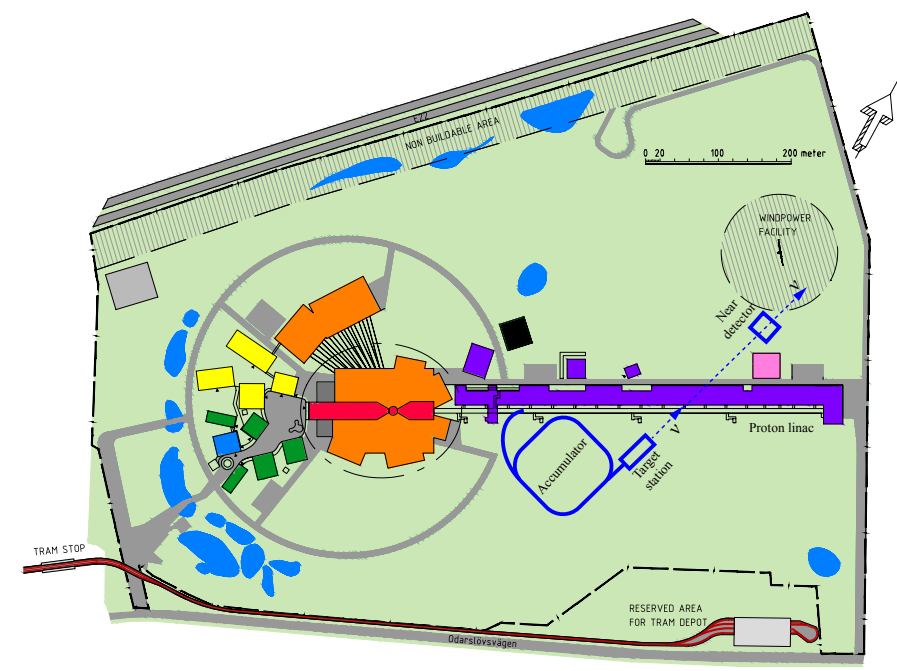

Figure 2: Schematic view of the ESS neutron and neutrino facility (in blue).

As can be seen by Fig. 3, presenting the neutrino spectrum which can be obtained at an arbitrary distance of $100 \mathrm{~km}$ for one year running, a small contamination of the muon neutrino beam from $0.5 \%$ electron neutrinos is expected. These neutrinos polluting the signal at the level of the far detector, as said above, could be used by a "smart" near detector to measure the electron neutrino cross-section at the neutrino energies used by the project and thus, help to further reduce the systematic errors.

For the voluminous far detector, a location, preferably near an active mine to decrease the excavation cost, has to be found. At a distance of $540 \mathrm{~km}$ from Lund, the active mine of Garpenberg 

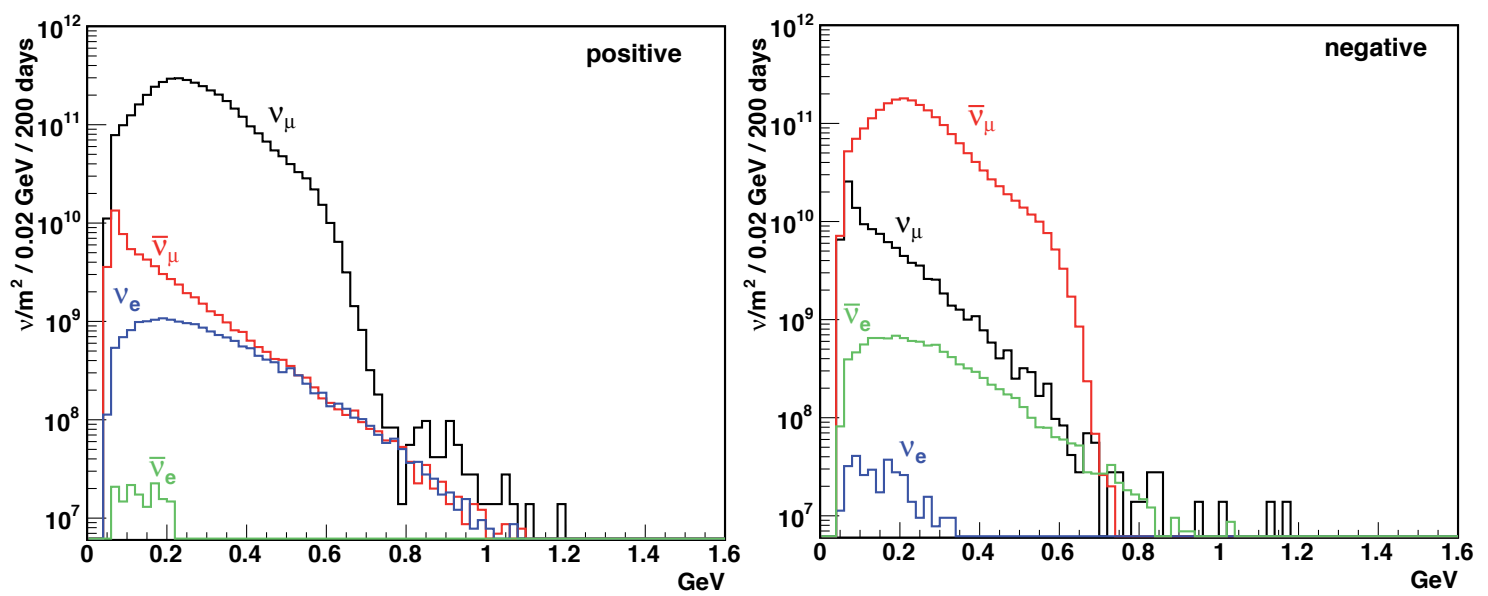

Figure 3: Neutrino energy spectrum for "neutrinos" (left, positive current in the horn) and "antineutrinos" (right, positive current in the horn) runs at a distance of $100 \mathrm{~km}$ on-axis from the target station, for $2.0 \mathrm{GeV}$ protons.

(Sweden) could host this megaton-class MEMPHYS-like Water Cherenkov detector [7].

\section{Physics Capabilities}

The Garpenberg mine, where the large Water Cherenkov detector could be installed, is located at a distance corresponding to the second maximum of the oscillation $v_{\mu} \rightarrow v_{e}$, taking into account the neutrino spectrum of Fig. 3. Fig. 4 presents the $v_{\mu} \rightarrow v_{e}$ oscillation probability as a function of the neutrino energy together with the neutrino energy distribution (in grey) of the detected by the far detector electron neutrinos coming from muon neutrino oscillations. It is clearly seen that the ESSvSB facility would be operated almost exclusively on the second oscillation maximum. Preliminary studies with limited optimisations show that ESSvSB could accumulate in 10 years data taking about 300 electron neutrinos (2 years running) and 250 electron antineutrinos ( 8 years running) coming from muon neutrino oscillations.

Fig. 5 presents the discovery significance as a function of the fraction covered of the CP violating parameter $\delta_{C P}$. For a proton energy of $2 \mathrm{GeV}$ (present design), 55\% of $\delta_{C P}$ can be covered with a $5 \sigma$ significance, while for a proton energy of $2.5 \mathrm{GeV}$ (linac upgrades) this fraction goes higher than $60 \%$. The comparison with other facilities is done assuming an unknown mass hierarchy and $5 \%$ systematic error on signal and $10 \%$ on background. The obtained $\delta_{C P}$ coverage by ESS $v$ SB facility is limited by the statistics and not by systematics. Of course, increasing the data taking period would increase the $\delta_{C P}$ coverage.

The ESS $v$ SB facility together with the neutrino production would also produce a huge number of muons. These muons could be collected at the level of the beam dump and used for other applications. The mean momentum of the muons is of the order of $0.5 \mathrm{MeV}$. A collecting device could be placed at the level of the beam dump to deviate and collect all these muons. More than $4 \times 10^{20}$ muons per year can be extracted. These muons can be used for a "low" nuSTORM neutrino experiments [8], for a Neutrino Factory and for R\&D for 6D muon cooling and studies for a possible future muon collider. 


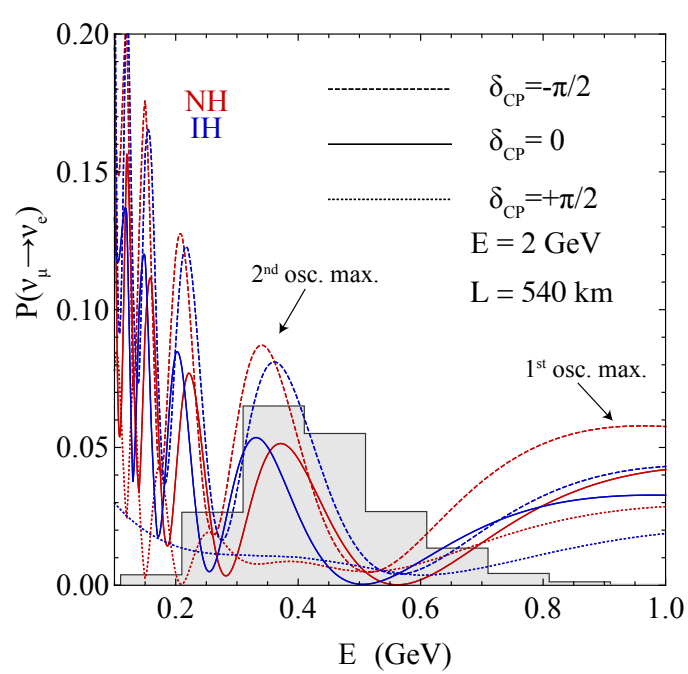

Figure 4: $\quad v_{\mu} \rightarrow v_{e}$ oscillation probability as a function of the neutrino momentum. The shaded distribution is the momentum distribution of electron neutrinos as they would be detected by the far detector.

\section{Aknowledgements}

This project is supported by the COST Action CA15139 "Combining forces for a novel European facility for neutrino-antineutrino symmetry-violation discovery" (EuroNuNet).

\section{References}

[1] S. Pascoli, S. T. Petcov and A. Riotto, Nucl. Phys. B 774 (2007) 1 doi:10.1016/j.nuclphysb.2007.02.019 [hep-ph/0611338].

[2] "A Very Intense Neutrino Super Beam Experiment for Leptonic CP Violation Discovery based on the European Spallation Source Linac", Nuclear Physics B, Volume 885, August 2014, 127-149.

[3] The European Spallation Source, http://europeanspallationsource.se/, ESS TDR, Release 1.0, Nov. 2012.

[4] “Optimization of neutrino oscillation facilities for large $\theta_{13}$," JHEP 1204, 089 (2012).

[5] T. R. Edgecock, O. Caretta, T. Davenne, C. Densham, M. Fitton, D. Kelliher, P. Loveridge and S. Machida et al., "The EUROnu Project,” Phys. Rev. ST Accel. Beams 16, 021002 (2013) [arXiv:1305.4067 [physics.acc-ph]].

[6] E. Baussan et al. [EUROnu Super Beam Collaboration], "The SPL-based Neutrino Super Beam," Phys. Rev. ST Accel. Beams 17, 031001 (2014), arXiv:1212.0732 [physics.acc-ph].

[7] "Study of the performance of a large scale water-Cherenkov detector (MEMPHYS)," JCAP 1301 (2013) 024.

[8] "nuSTORM - Neutrinos from STORed Muons: Letter of Intent to the Fermilab Physics Advisory Committee," arXiv:1206.0294 [hep-ex]. 